\title{
Phylogenetic Reconstruction with Gene Rearrangements and Gene Losses
}

\author{
Yiwei Zhang, Fei Hu and Jijun Tang \\ Department of Computer Science and Engineering \\ University of South Carolina \\ Columbia SC, USA \\ Email: \{zhang33,hu5,jtang\}@cec.sc.edu
}

\begin{abstract}
Reconstructing phylogenies from gene-order data has become very attractive in the research of evolution these years. So far, most methods can only treat genomes with equal gene contents with each gene appearing exactly once in each genome. In this paper, we propose a new distance measurement for genomes with inversions and insertions/deletions that comply with triangle inequality. Based on this distance, we develop a new method to solve the median problem of unequal gene content, which are used to reconstruct both phylogenies and ancestral genomes. We test our method on simulated datasets under various conditions and the experimental results show that our distance measurement can produce more accurate phylogenetic trees compared with other popular methods for unequal genomes. Also our median algorithm produces remarkably more accurate ancestral genomes than the only unequal genome median solver that is currently available.
\end{abstract}

Keywords-Phylogeny; Genome Rearrangement; Gene Loss; Branch-and-bound;

\section{INTRODUCTION}

High-level rearrangement events such as inversions, transpositions that change gene order are important. Because rearrangements of genes are "rare genomic events", these orderings enable the reconstruction of evolutionary events far back in time [3]. Genome rearrangement analysis can also be used to estimate ancestral genomes and infer the number of steps that evolve one genome into another, therefore it is important and has a wide range of applications.

Much progress has been made in analyzing genome rearrangements. However, most methods assume equal gene content, i.e., each gene appears exactly once in each given genomes. Such assumption is far from realistic since gene deletions and insertions are common. Various methods have been proposed to compare two genomes with unequal gene content, but very few are able to both reconstruct phylogenies and infer ancestral genomes. Indeed, to our knowledge, the method developed by Tang and Moret [5] might be the only publicly available tool that can conduct both tasks, although it is far from satisfactory.

In this paper, we propose a new distance model that complies with triangle inequality for genomes with unequal gene content. Also, we present a median solver algorithm based on our distance measurement, apply these methods to reconstruct phylogeny and estimate ancestral genomes. Extensive experiments are conducted on simulated datasets and the results show that our new distance measurement is more accurate compared with other current approaches and our median solver finds more accurate ancestral genomes.

\section{BACKGROUNDS}

\section{A. Genome Rearrangements}

Given a set $S$ of $n$ genes $\{1,2, \cdots, n\}$, a genome can be represented by an ordering of these genes. A gene with a positive orientation is written as $i$, otherwise it is written as a $-i$. A genome can be linear or circular. Let $G$ be the genome with signed ordering of $1,2, \cdots, n$. An inversion (also called reversal) between indices $i$ and $j(i \leq j)$, transforms $G$ to a new genome with ordering

$$
1,2, \cdots, i-1,-j,-(j-1), \cdots,-i, j+1, \cdots, n
$$

An insertion is the addition of one gene or a segment of genes, and a deletion is the loss of a section of the chromosome.

We use breakpoint graph [1] to represent the permutation with respect to the identity permutation. Given a genome with permutation $\pi$, let the breakpoint graph corresponding to it be $M(\pi)$. The vertex set $V$ of $M(\pi)$ is the collection of $\{2 i-1,2 i\}$, and $i$ is any distinct gene of permutation $\pi$. Two genes $i$ and $j$ are adjacent in genome $G$ if $i$ is immediately followed by $j$ and can be represented by an edge $(2 i, 2 j$ 1). If $i$ and $-j$ are adjacent, then it can be represented by an edge $(2 i, 2 j)$. The edge set $E$ of $M(\pi)$ consists of all the adjacencies in $\pi$. For example, for a circular unichromosomal genome $G_{2}=1,4,2,-3,5$, the vertex set is $V=\{1,2,3,4,5,6,7,8,9,10\}$ and the edge set is $E=$ $\{(10,1),(2,7),(3,8),(4,6),(5,9)\}$, as shown in Fig. 1.

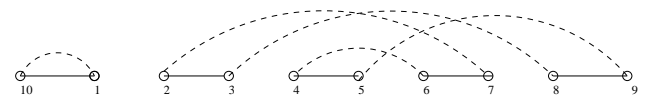

Figure 1. Breakpoint graph of genome $G=1,2,-3,4,-5$, with respect to the identity genome $G=1,2,3,4,5$

\section{B. Distance Computation}

The inversion distance between two genomes is the minimum number of inversions needed to transform one genome into another. Given two permutations $\pi_{1}, \pi_{2}$, the set of edges 
of $M\left(\pi_{1}\right)$ and $M\left(\pi_{2}\right)$ defines a set of cycles whose edges are alternately in $M\left(\pi_{1}\right)$ and $M\left(\pi_{2}\right)$. Let $n$ be the length of the unichromosomal genome and $c\left(\pi_{1}, \pi_{2}\right)$ denote the number of cycles, we will call $n-c\left(\pi_{1}, \pi_{2}\right)$ the cycle distance, which is a tight lower bound of the inversion distance [1].

A double cut and join DCJ operation [6] swaps two gene ends in two different vertices of the same genome in the adjacency graph. The DCJ operation is proposed to account for all rearrangement events and it provides a simpler and unifying model for genome rearrangement. DCJ distances is defined as the number of minimal DCJ operations needed to transform one permutation into another and it is equivalent to the cycle distance.

Both inversion and DCJ distances defined above can only handle genomes with equal gene contents. For unequal genomes with gene insertions/deletions, El-Marbrouk [2] proposed a definition of the distance between such genomes based on the assumption that the cost of deleting one continuous block of genes is equal to that of one inversion operation. This implies that deleting blocks with more genes is always preferred. But this distance definition cannot comply with triangle inequality. For example, if $\pi_{1}=1,2,3,4,5$, $\pi_{2}=1,4,2,-3,5, \pi_{3}=1,5$, then $d\left(\pi_{1}, \pi_{2}\right)=3, d\left(\pi_{1}, \pi_{3}\right)=$ $1, d\left(\pi_{2}, \pi_{3}\right)=1$, so $d\left(\pi_{1}, \pi_{2}\right)>d\left(\pi_{1}, \pi_{3}\right)+d\left(\pi_{2}, \pi_{3}\right)$, which cannot satisfy triangle inequality.

\section{Median Problem and Phylogenetic Reconstruction}

The core of the tools that reconstruct genome rearrangement phylogenies remains a set of algorithms to solve the median problem, which is defined as follows: given a set of $N$ genomes with permutations $\left\{\pi_{i}\right\}_{1 \leq i \leq N}$ and a distance measure $d$, find a permutation $\pi_{M}$ that minimizes the median score $\sum_{i=1}^{N} d\left(\pi_{i}, \pi_{M}\right)$. In phylogenetic practice, since we always examine binary trees, the value of $N$ is three. The median problem is known to be NP-hard [1].

Tang and Moret improved Siepel's solver by adding new bounds and procedures to determine the ancestral gene contents, producing a method that can handle genomes when both inversions and deletions (or insertions) are present [5].

\section{Distance COMPUTATION FOR GENOMES With UNEQUAL CONTENT}

For genomes with inversions and gene insertions/deletions, we propose a distance model based on DCJ distance. Contrary to the model used by ElMarbrouk, we use a simplified model and assume the cost of insertion/deletion of each gene to be one.

\section{A. New Model of Distance Computation}

Given two circular unichromosomal genomes with permutation $\pi_{1}$ and $\pi_{2}$, we define the distance as follows:

$$
d\left(\pi_{1}, \pi_{2}\right)=m-c\left(\pi_{1}^{\prime}, \pi_{2}^{\prime}\right)+\left(n_{1}-m\right)+\left(n_{2}-m\right)
$$

In this definition, $m$ is the number of genes that appear both in $\pi_{1}$ and $\pi_{2}$; the length of $\pi_{1}$ is $n_{1}$ and the length of $\pi_{2}$ is $n_{2}$. So $n_{1}-m$ is the number of genes that appear in $\pi_{1}$ but not included in $\pi_{2} ; n_{2}-m$ is the number of genes that appear in $\pi_{2}$ but not included in $\pi_{1}$. Let $\pi_{1}^{\prime}$ be the reduced permutation of $\pi_{1}$ which removes the genes not in $\pi_{2}$. Let $\pi_{2}^{\prime}$ be the reduced permutation of $\pi_{2}$ which removes the genes not in $\pi_{1}$. We can see that $m-c\left(\pi_{1}^{\prime}, \pi_{2}^{\prime}\right)$ corresponds to the DCJ distance between the reduced genomes and $\left(n_{1}-m\right)+$ $\left(n_{2}-m\right)$ corresponds to the cost of unequal genes.

For example, if genome $G_{1}$ has the permutation 1,2, 3 and genome $G_{2}$ has the permutation -3,-2, 4, they have genes 2,3 in common and two different genes 1,4 . After removing the unequal genes, the reduced permutations become 2,3 and -3, -2 and the DCJ distance between these reduced permutations is 1 . Altogether the distance between $G_{1}$ and $G_{2}$ is $2+1=3$.

\section{B. Triangle Inequality}

We can prove that our defined distance complies with triangle inequality. For any three genomes with permutation $\pi_{1}, \pi_{2}$ and $\pi_{3}$, our distance definition satisfies that

$$
d\left(\pi_{1}, \pi_{2}\right) \leq d\left(\pi_{1}, \pi_{3}\right)+d\left(\pi_{2}, \pi_{3}\right)
$$

Proof: Let $n_{i}$ denote the length of permutation $\pi_{i}$. Let $n_{i j}$ denote the number of common genes between $\pi_{i}$ and $\pi_{j} . \pi_{i}^{j}$ is the reduced permutation of $\pi_{i}$ which removes the genes not present in $\pi_{j}$. First, let us consider the case that $\pi_{3}$ contains all the common genes between $\pi_{1}$ and $\pi_{2}$. Also $\pi_{3}$ does not contain genes that are neither present in $\pi_{1}$ nor in $\pi_{2}$. In this way we can see that $n_{13} \geq n_{12}$ and $n_{23} \geq n_{12}$. $\pi_{3}^{12}$ is the reduced permutation of $\pi_{3}$ which only contains the genes that appear in both $\pi_{1}$ and $\pi_{2}$. According to the definition we have:

$$
\begin{gathered}
d\left(\pi_{1}, \pi_{3}\right)+d\left(\pi_{2}, \pi_{3}\right)-d\left(\pi_{1}, \pi_{2}\right)= \\
2 n_{3}-c\left(\pi_{1}^{3}, \pi_{3}^{1}\right)-n_{13}-c\left(\pi_{2}^{3}, \pi_{3}^{2}\right)-n_{23}+n_{12}+c\left(\pi_{1}^{2}, \pi_{2}^{1}\right)
\end{gathered}
$$

Because the number of cycles is no greater than the length of permutation, we have $c\left(\pi_{1}^{3}, \pi_{3}^{1}\right) \leq n_{13}-n_{12}+c\left(\pi_{1}^{2}, \pi_{3}^{12}\right)$ and $c\left(\pi_{2}^{3}, \pi_{3}^{2}\right) \leq n_{23}-n_{12}+c\left(\pi_{2}^{1}, \pi_{3}^{12}\right)$, we can rewrite the above equation and get:

$$
\begin{gathered}
d\left(\pi_{1}, \pi_{3}\right)+d\left(\pi_{2}, \pi_{3}\right)-d\left(\pi_{1}, \pi_{2}\right) \geq \\
2\left(n_{3}-n_{13}-n_{23}+n_{12}\right)+n_{12}-c\left(\pi_{1}^{2}, \pi_{3}^{12}\right)+n_{12}- \\
c\left(\pi_{2}^{1}, \pi_{3}^{12}\right)-\left(n_{12}-c\left(\pi_{2}^{1}, \pi_{1}^{2}\right)\right)
\end{gathered}
$$

According to [1], we have

$n_{12}-c\left(\pi_{1}^{2}, \pi_{3}^{12}\right)+n_{12}-c\left(\pi_{2}^{1}, \pi_{3}^{12}\right)-\left(n_{12}-c\left(\pi_{2}^{1}, \pi_{1}^{2}\right)\right) \geq 0$

Also we have $n_{3}+n_{12}-n_{13}-n_{23}=0$, so we can get $d\left(\pi_{1}, \pi_{3}\right)+d\left(\pi_{2}, \pi_{3}\right)-d\left(\pi_{1}, \pi_{2}\right) \geq 0$

Next let us consider the case that $\pi_{3}$ does not contain all the genes that are both present in $\pi_{1}$ and $\pi_{2}$. In this case let $\pi_{3}^{\prime}$ be the expanded permutation that includes all the genes present in $\pi_{1}$ and $\pi_{2}$. Because of the extra insertion operations of genes, we have $d\left(\pi_{1}, \pi_{3}\right) \geq d\left(\pi_{1}, \pi_{3}^{\prime}\right)$, 
$d\left(\pi_{2}, \pi_{3}\right) \geq d\left(\pi_{2}, \pi_{3}^{\prime}\right)$ So we have $d\left(\pi_{1}, \pi_{3}\right)+d\left(\pi_{2}, \pi_{3}\right) \geq$ $d\left(\pi_{1}, \pi_{3}^{\prime}\right)+d\left(\pi_{2}, \pi_{3}^{\prime}\right) \geq d\left(\pi_{1}, \pi_{2}\right)$.

Finally we can prove the case that $\pi_{3}$ contains genes that are neither present in $\pi_{1}$ nor $\pi_{2}$ in the same way.

\section{Algorithm for Unequal Genome Median PROBLEM}

In this section, we extend Caprara's branch-and-bound median algorithm to calculate medians for unequal genomes, using our new distance measurement. Our new method does not guess which gene appears in the median genome, as the content will be determined solely on whether or not adding a gene can lower the median score.

Similar to Caprara's algorithm, we iteratively search and expand a tree until the median solution is found. For any tree node that corresponds to a fixed adjacency $(i, j)$, we proceed at this node with the enumeration by fixing $(j+1$, $k$ ) (if $j$ is odd) or $(j-1, k$ ) (if $j$ is even), for all $k$ that is not within the adjacencies from the root to the current tree node. The lower bound of a node is the optimal median score that might be achieved if all the adjacencies from root to current node are included. We always search and expand the leave nodes whose lower bounds are minimum until the median score of a leave equals to minimum lower bound.

Because our distance measurement complies with the triangle inequality, according to [1], given three permutations $\pi_{1}, \pi_{2}$ and $\pi_{3}$, the lower bound on the median score is $\left\lceil\frac{d\left(\pi_{1}, \pi_{2}\right)+d\left(\pi_{1}, \pi_{3}\right)+d\left(\pi_{2}, \pi_{3}\right)}{2}\right\rceil$.

We calculate the lower bound with fixed adjacencies as follows. Given a genome with permutation $\pi$, let the MBG that corresponds to it be $M(\pi)$. According to [1], given an edge $e$, the median containing $e$ is the median of the permutations obtained by contracting edge $e$. For an edge $(i, j)$, if the vertex set $V$ already contains both $i$ and $j$, the contraction of e can be performed as [1] describes. If $i$ is within vertex set $V$ but $j$ is not in $V$, then let $(i, a)$ be the edge corresponding to $i$. The contraction of $(i, j)$ would remove edge $(i, a)$ and create a new edge $(a, j+1)$ if $j$ is odd or $(a, j-1)$ if $j$ is even. This is the case only happens for unequal genomes. So the lower bound $l b$ of the median score with fixed edge $e$ is:

$$
l b=l b^{\prime}+\sum_{i=1}^{N} d_{i}
$$

here $l b^{\prime}$ is the lower bound of the new permutations obtained by contracting edge $e$ from original permutations. If $e$ is an instance within the edge set of $M(\pi)$, then the value of $d_{i}$ is 0 , otherwise it is 1 .

\section{EXPERIMENTAL RESULTS}

\section{A. Robustness of Distance Model}

We conduct simulations to determine the rationality of our proposed unequal genome distance measurement by examining the topological correctness of reconstructed phylogenetic trees. Let $T$ be the true tree and $T^{\prime}$ be the inferred phylogeny.
The falsenegatives $(F N)$ of $T^{\prime}$ with respect to $T$ are those edges present in true tree but not present in the inferred tree $T^{\prime}$. The falsepositives $(F P)$ are the edges present in $T^{\prime}$ but not present in $T$. Accordingly, the falsenegativesrate is the number of false negatives divided by the number of internal edges in $T$ and similarly we also have falsepositiverate. The Robinson-Foulds (RF) rate is defined as the average of the FN and FP rates.

We generated model tree topologies from uniform distribution on binary trees, each with 10 leaves. We chose genome sizes of 100 and 1000 genes. We use various numbers of evolutionary rates, with $4,8 \cdots 20$ expected events per tree edge. Of all the events, $80 \%$ are inversions and $20 \%$ are losses of one segment of genes. To test the robustness of our distance model, we used various upper bounds for the length of the lost segment: 3, 5 for genomes with sizes of 100, and 20, 40 for genomes with sizes of 1000 . We used our distance measurement to calculate the distance between genomes and the reconstructed phylogenetic trees using FastME. For each combination of parameters, we ran 100 data sets and averaged the results. We also use Swenson's method [4], which is a popular method to estimate the edit distance between unequal genomes to conduct the comparison of accuracy.

Fig. 2 shows the result of topological accuracy comparison, using genomes with sizes of 100 . When events are fewer than 8 per edge, the RF rates of both methods are very low. When events are between 8 and 12 per edge, the RF rate of Swenson's method increases dramatically while our method still holds very low RF rates. When events are over 12 per edge, the RF rates of both methods become higher, but still our method has smaller RF rate compared with Swenson's method.
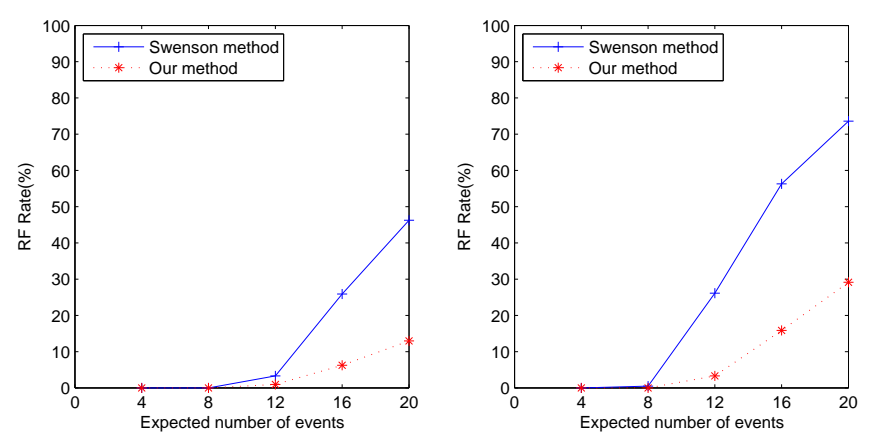

Figure 2. Maximum deletion length is 3 on the left and 5 on the right

Fig. 3 shows the result of the topological accuracy comparison, using genomes with sizes of 1000 . We can see similar result as above. When events are fewer than 120 per edge, the RF rates of both methods are very low. When events are over 120 per edge, RF rates of both methods become quite high, but our method has much lower RF rates than Swenson's method. 

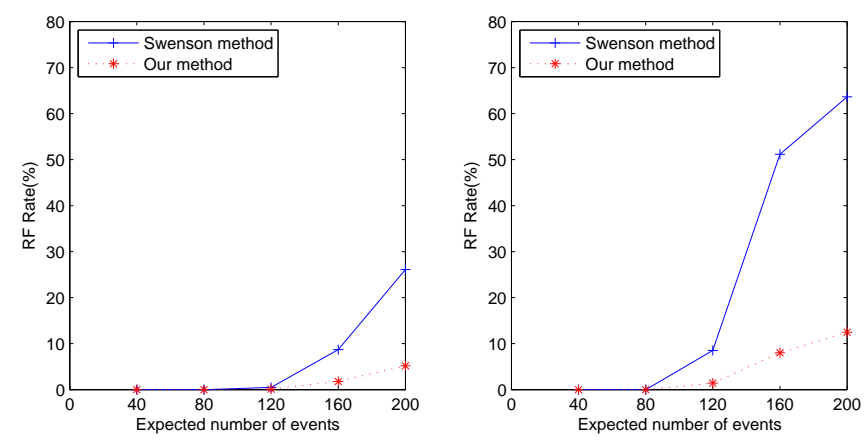

Figure 3. Maximum deletion length is 20 on the left and 40 on the right

\section{B. Phylogeny and Ancestral Genome Accuracy}

We implemented our median algorithm in the GRAPPA framework and obtained a new phylogenetic reconstruction method and compared it with Tang and Moret's method [5]. We chose datasets of $6,8,10,12$ genomes with sizes of 100 genes and evolutionary rates of 10 expected events per tree edge. Of all the events, $80 \%$ are inversions and $20 \%$ are losses of one segment of genes. The upper bound of the length of lost segment is 4. For each combination setting, we ran 30 data sets and average the results.

Fig. 4 shows the RF rates of both methods. Clearly our new method is more accurate than the previous one. For 12 genomes, our method reconstructed completely true trees on $90 \%$ datasets, while the previous method only achieved $78 \%$.

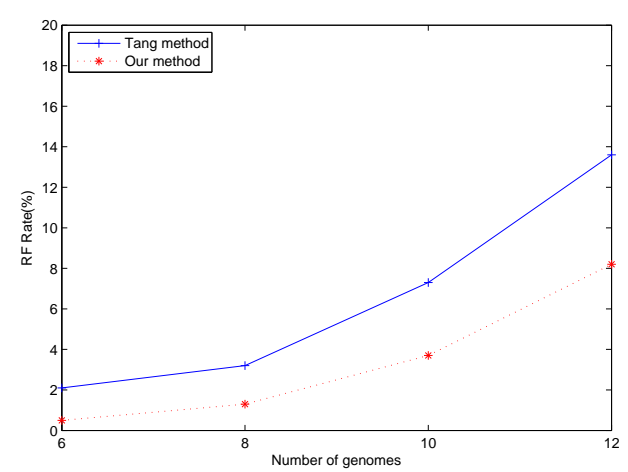

Figure 4. RF rate comparison of the new median solver

We compared the inferred ancestral genomes to the true one when both methods reconstruct the true trees. The correctness of the reconstructed ancestral genome is measured by checking gene adjacency. Let $G$ be the permutation of the true genome and $G^{\prime}$ be the permutation of the genome that we constructed. Then the false negatives of $G^{\prime}$ with respect to $G$ are those adjacencies present in $G$ but not present in the inferred permutation $G^{\prime}$. The false positives of $G^{\prime}$ with respect to $G$ are the adjacencies present in inferred $G^{\prime}$ but not in $G$. Accordingly, the false negatives rate is the number of false negatives divided by the number of adjacencies of $G$ and similarly we have false positive rate. We define adjacency error rate as the average of the FN and FP rates. The following table shows the adjacency error rate of our method compared with Tang and Moret's method. We can see that with the increase of genome numbers, the error rate of our method increases slightly but is far lower than original method. This suggests that the gene content prediction of their method is not optimal. Such prediction is not necessary since the content can be determined along with the determination of optimal median gene order.

\begin{tabular}{|c|c|c|c|c|}
\hline \# of genomes & 6 & 8 & 10 & 12 \\
\hline Our method & 0.0161 & 0.0234 & 0.0381 & 0.0536 \\
\hline Tang's method & 0.3165 & 0.3196 & 0.3758 & 0.3840 \\
\hline
\end{tabular}

COMPARISON OF ADJACENCY ERROR RATES OF INTERNAL PERMUTATION WITH DIFFERENT GENOME SIZES

\section{CONCLUSIONS}

In this paper we proposed a distance measurement for genomes with gene inversions and insertions/deletions. We also developed a branch-and-bound algorithm to solve the median problem based on our distance approach. The simulation test is performed with various cases including small continuous genes deletion and large continuous genes deletion. Our experimental results show that this new distance measurement is more accurate for reconstructing phylogenetic trees compared with other methods. Also the reconstructed genomes of internal nodes by our median algorithm are more accurate than previous methods.

\section{ACKNOWLEDGEMENTS}

This work is partly supported by grants NSF 0904179 and NIH GM078991-03S1.

\section{REFERENCES}

[1] Caprara, A. (2003). The reversal median problem. INFORMS Journal on Computing, 15(1), 93-113.

[2] El-Marbrouk, N. (2000). Genome rearrangement by reversals and insertions/deletions of contiguous segment. In Proc. 11th Ann. Symp. Combinational Pattern Matching, volume 1848 of Lecture Notes in Computer Science, 222-234.

[3] Sankoff, D. and M. Blanchette (1998). Multiple genome rearrangement and breakpoint phylogeny. J. Comput. Biol. $5,555-570$.

[4] Swenson, K., W. Arndt, J. Tang and B. More (2008). Phylogenetic reconstruction from complete gene orders of whole genomes. Sixth Asia Pacific Bioinformatics Conference (APBC 2008), in Advances in Bioinformatics and Computational Biology Vol. 6, 241-250.

[5] Tang, J., B. Moret, L. Cui, and C. dePamphilis (2004) Phylogenetic reconstruction from arbitrary gene-order data. Proc. 4th IEEE Conf. on Bioinformatics and Bioengineering (BIBE 2004) 592-599.

[6] Yancopoulos S., Attie O., and Friedberg R. (2005). Efficient sorting of genomic permutations by translocation, inversion and block interchange. Bioinformatics, 21, 3340-3346. 